\title{
ENSEÑANZA EFICAZ DE LA RESOLUCIÓN DE PROBLEMAS EN MATEMÁTICAS
}

\author{
María Mayela Calvo Ballestero \\ Estudiante de la Maestría en Planificación Curricular \\ Universidad de Costa Rica \\ San José, Costa Rica
}

Recibido: 27-XI-2007 • Aceptado 11-III-2008 • Corregido 20-IV-2008

\begin{abstract}
Resumen: El presente artículo surge debido a la preocupación existente a causa del bajo rendimiento en matemática, factor que ha sido causante de la deserción y repitencia en el sistema educativo costarricense. La resolución de problemas ha sido considerada una de las áreas de la matemática que mayor dificultad ha presentado para la población estudiantil. Los niños y las niñas son capaces de resolver mecánicamente las operaciones fundamentales básicas (suma, resta, multiplicación y división), pero no saben cómo aplicarlas para la solución de un problema, ya que sólo se les ha enseñado a actuar de forma mecánica y repetitiva, por ello es fundamental tomar conciencia acerca de la problemática vivida en torno a este tema, y a su vez tomar las medidas necesarias para lograr el mejoramiento en los procesos de enseñanza y aprendizaje de la resolución de problemas.
\end{abstract}

Palabras clave: Resolución de problemas, enseñan$z a$, aprendizaje, matemática.

\section{Introducción}

A pesar de que se han realizado grandes esfuerzos por mejorar la calidad y cobertura del sistema educativo, la deserción y el rezago escolar continúan; actualmente la preocupación crece, dado que los índices de reprobación en lugar de disminuir, aumentan; de acuerdo con el Estado de la Educación Costarricense (Programa Estado de la Nación en Desarrollo Humano Sostenible, 2005, p. 26).

La reprobación incrementa la probabilidad de deserción del sistema educativo, tiene efectos psicológicos adversos en los estudiantes reprobados y aumenta el número de años que necesita la población estudiantil para graduarse, lo que representa un costo financiero adicional para el Estado y las familias.

El Estado de la Educación Costarricense, (Programa Estado de la Nación en Desarrollo Humano Sostenible, 2005) muestra algunas percepciones de los niños y las niñas que repiten la educación primaria, entre las principales razones que mencionan se encuentran: la falta de esfuerzo por parte de los estudiantes, estudios escolares poco interesantes, lo cual genera 


\begin{abstract}
This article comes from the current concern about the students' low performance in mathematics, as a factor that has caused many of them to drop the school and repeat the same grade. Problem solving has been considered one of the most difficult areas of mathematics for students. Even though boys and girls are able to solve mechanically basic arithmetic operations (addition, subtraction, multiplication, and division), they do not know how to apply them for solving problems. This is because students have been taught to act in a mechanical and repetitive way. Therefore, it is important to make people conscious about this situation of the Costa Rican educational system. In addition, specific actions must be taken in order to improve the teaching-learning processes of the problem solving
\end{abstract}

Key words: Problem solving, teaching, learning, math. falta de atracción hacia ellos y dificultad al realizar los deberes escolares.

En el contexto educativo costarricense, una de las materias de mayor índice de reprobación es la matemática, en el desarrollo de esta asignatura ha predominado un enfoque curricular academicista, el cual según explica Castillo (2003), se caracteriza por la transmisión de conocimiento del docente hacia el estudiante, quien se considera el protagonista por poseer el saber, esta actitud genera que los alumnos y las alumnas tengan un rol pasivo en los procesos de enseñanza y aprendizaje.

Una de las áreas de la matemática que mayor dificultad adquiere para los estudiantes y las estudiantes es la resolución de problemas; los niños y las niñas son capaces de resolver mecánicamente las operaciones fundamentales básicas (suma, resta, multiplicación y división), pero no saben cómo aplicarlas para la solución de un problema, ya que sólo se les ha enseñado a actuar de forma mecánica y repetitiva. Según Kamii (1994), citado por Ruiz y otros (2003, p. 326) “...La resolución de problemas debería darse al mismo tiempo que el aprendizaje de las operaciones en vez de después, como aplicaciones de éstas..."; por lo tanto, el aprendizaje simultáneo de ambos facilitaría la comprensión y asimilación de las operaciones aritméticas.

La metodología empleada en la enseñanza de la resolución de problemas en matemáticas, es un elemento clave para el logro satisfactorio de los contenidos en esta área, Polya (1965), citado por Echenique (2006, p. 10), explica “...que el profesor tiene en sus manos la llave del éxito ya que, si es capaz de estimular en los alumnos la curiosidad, podrá despertar en ellos el gusto por el pensamiento independiente; pero, si por el contrario dedica el tiempo a ejercitarles en operaciones de tipo rutinario, matará en ellos el interés...". Los estudiantes y las estudiantes deben ser introducidos de forma agradable con actividades que mantengan el interés en la materia y evite abstracciones que conllevan a la desmotivación 
ante la falta de comprensión de los diversos conceptos.

Es necesario un cambio para erradicar la concepción de la matemática como una materia aburrida y difícil, se debe tomar conciencia acerca de la problemática vivida en torno a este tema, pero también es necesario tomar las medidas necesarias para lograr el mejoramiento en el proceso de enseñanza-aprendizaje de la resolución de problemas.

Debido a lo anterior, se presentarán aspectos referentes a las dificultades que muestran los estudiantes con respecto al aprendizaje de la matemática, la metodología que se debe emplear para obtener una enseñanza eficaz en relación al tema y que promueve el desarrollo de la inteligencia lógico matemática en los niños y las niñas de educación primaria.

Se considera que para enseñar la resolución de problemas en matemática se debe aplicar una metodología que ayude al estudiante a hallar la solución correcta de una manera comprensiva; para lograr esto es importante reconocer aspectos referentes al papel del docente y del alumno en este proceso, así como la influencia que tiene la actitud que muestren ambos sujetos.

En el siguiente apartado se presentan los fundamentos teóricos que sustentan este artículo, los cuales corresponden a investigaciones realizadas que han permitido mejorar en cierta medida el trabajo realizado por el personal docente en las clases de matemática.

\section{Fundamentos teóricos}

Terán y otros (2005), explicaron que las clases de matemática inician a partir de la definición de contenidos carentes de significados para estudiantes de niveles de educación básica, ya que por lo general se alejan de sus vivencias. Como consecuencia de ello, se les dificulta reconocer la importancia de la matemática y los lleva a preguntarse ¿para qué sirve esta materia?
En torno a esta problemática, se han realizado algunos trabajos cuyos aportes se consideran valiosos para el área en estudio, entre ellas se pueden mencionar las siguientes:

A) Pinteño y otros (1999) estudiaron la mejora del rendimiento en el área de matemáticas a través de la resolución de problemas con alumnado de educación primaria. Esta investigación tiene como base estudios realizados en Estados Unidos y España, los cuales muestran resultados mediocres o muy bajos en cuanto a la resolución de problemas de dos operaciones por parte de los estudiantes de la escuela elemental y media de estos países.

Con la investigación se tenía la pretensión de lograr una mejora sustancial de los procesos de enseñanza y aprendizaje en el área de matemáticas aplicando un modelo de resolución de problemas.

Cualquier sesión sigue este esquema general de trabajo:

a. Introducción por parte de la persona instructora con los componentes manipulativos.

b. Explicación de los componentes gráficos y simbólicos.

c. Realización por parte de los sujetos de los problemas. Esta tarea fue realizada individualmente, en parejas o en pequeños grupos.

d. Corrección de la tarea, cuando la mayoría del grupo había terminado el trabajo. Se promovió la discusión de las soluciones aportadas por el alumnado, lo cual favorece a la creación de un conflicto cognitivo en el caso de la existencia de soluciones divergentes. Se hace especial hincapié en la comprobación del resultado volviendo a leerse la pregunta del problema y comprobando si la solución aportada se corresponde con lo pedido. Se tuvo especial cuidado en el tratamiento de los errores cometidos por el 
alumnado, tratando de considerarlos como algo natural durante el proceso de enseñanza-aprendizaje.

En los resultados se logró conocer que la aplicación del programa instruccional en resolución de problemas aritméticos a un grupo de alumnos y alumnas muestra resultados sensiblemente superiores en las puntuaciones finales, respecto a las iniciales en las diversas categorías semánticas de problemas.

B) Sánchez (2001) realizó una investigación acerca de las dificultades de los alumnos de sexto grado de educación primaria para la resolución de problemas matemáticos. Esta se adentra en un grupo de alumnos que mostraron dificultad en esta área, se hizo un análisis retrospectivo de las enseñanzas y aprendizajes a las cuales han sido sujetos a lo largo de su educación primaria. Desentrañó así que las dificultades para no resolver correctamente los problemas, no radican en el alumno mismo, sino que entran otros aspectos en juego, tales como la metodología empleada por el docente o la actitud que éste tenga hacia la materia.

El objetivo principal de la investigación fue conocer y comprender la relación que existe entre las dificultades para la resolución de problemas matemáticos presentes en los alumnos de sexto grado y la forma en cómo les enseñaron las matemáticas en los grados anteriores, y así es posible estar en condiciones de establecer correlaciones entre ambos aspectos.

De acuerdo a los resultados se evidencia que la presencia de dificultades se debe a que no se tomó en cuenta durante su enseñanza, la maduración psicogenética, se ha olvidado, ignorado o desconocido que la concepción y comprensión por parte del estudiante de los contenidos matemáticos están en relación con el nivel de desarrollo en que se encuentre.

Según la investigadora, no se da un seguimiento lógico y continuo entre los elementos del proceso de enseñanza, en múltiples ocasiones se empieza por lo último, es decir, la ejercitación de mecanizaciones para luego aplicarlas a la resolución de problemas.

C) Ruiz y otros (2003, p. 321) realizaron una investigación llamada "El lenguaje como mediador de la aritmética en la primera etapa de educación básica". Este trabajo tuvo como fundamento la perspectiva constructivista de Piaget y la corriente históricocultural propuesta por Vigotsky. Su propósito fue diseñar, ejecutar y evaluar estrategias didácticas para promover el desarrollo del pensamiento aritmético, utilizando el lenguaje como mediador en niños y niñas de la primera etapa de educación básica de la escuela rural Mirabelito de Trujillo en México.

Se retoman algunas ideas importantes acerca de las mejores formas para enseñar los conceptos numéricos en los primeros años de escolarización, entre las estrategias didácticas más sobresalientes en la investigación se encuentran:

- Promover la comunicación oral y escrita, como forma de hacer con las palabras las mismas acciones que se hacen con los objetos; en este sentido, la promoción de la aritmética oral debe ser tan importante como la escrita.

- Propiciar el desarrollo de la "reversibilidad", como estrategia cognitiva, mediante la cual en la acción de "devolverse" debe lograrse la comprensión de las nuevas relaciones que aparezcan y de la forma diferente en que se manifiestan las acciones preliminares.

Tomando en cuenta lo anterior, se plantea la pertinencia de comenzar a 
transformar la práctica pedagógica y, por tanto, el aprendizaje de los conocimientos básicos mediante la perspectiva de la investigación-acción.

Los hallazgos evidenciaron que los niños y las niñas tratan de describir e interpretar el proceso de resolución de problemas, mostraron satisfacción por el trabajo cooperativo y desarrollo progresivo de la autonomía en la realización de las actividades escolares. De este análisis surgió una afirmación general, la cual se refiere a que cada estudiante es capaz de desarrollar conocimientos aritméticos $\mathrm{y}$ lingüísticos significativos cuando se promueven estrategias didácticas tales como: el juego, la resolución de problemas, la reversibilidad, la interacción verbal, además, la lectura y escritura fueron asumidas como procesos generadores de significados.

D) Terán y otros (2005) desarrollaron "La investigación-acción en el aula: tendencias y propuestas para la enseñanza de la matemática en sexto grado". Esta investigación tuvo como propósito determinar la aplicabilidad de un conjunto de estrategias constructivistas para la enseñanza y el aprendizaje de la matemática en sexto grado de la educación básica. La metodología utilizada fue la investigación-acción participativa, que implicó un trabajo de campo caracterizado por la observación y participación intensiva a largo plazo en una unidad educativa del estado de Trujillo. Se seleccionaron como categorías de análisis: la práctica pedagógica desarrollada por la maestra y el trabajo cooperativo.

Se menciona la necesidad de repensar la manera como se trabaja la matemática, la cual se imparte de manera mecánica y repetitiva. Este problema es inherente a todas las etapas del proceso educativo: planificación, ejecución y evaluación; por lo general se planifica en función del programa de estudio y no en función del alumno, lo cual hace que la materia no sea significativa para el estudiante.

El diseño y aplicación de estrategias metodológicas constructivistas para facilitar el aprendizaje, condujo a logros tanto para el grupo de estudiantes como para la maestra. En el alumnado permitió: desarrollar actitudes positivas tendentes a mejorar el aprendizaje de la matemática, formular, proponer e inventar nuevos problemas matemáticos, desarrollar un pensamiento crítico, crear y recrear el conocimiento matemático. De igual manera, se logró desarrollar en los niños y las niñas habilidades para el trabajo independiente y autónomo en la realización de actividades y consolidación de valores para la convivencia. En relación con la maestra, se consiguió mejorar su práctica pedagógica, optimizar, presentar situaciones reales o simuladas que permitieron a los niños y las niñas asumir actitudes reflexivas relacionadas con la construcción de conceptos matemáticos y, perfeccionar su capacidad creativa para diseñar otras estrategias metodológicas tendentes a mejorar el aprendizaje de la matemática.

Cada una de las investigaciones citadas, surge ante una necesidad evidente de mejora de los procesos de enseñanza y aprendizaje de la matemática, en los cuales hasta el momento no han sido utilizadas estrategias didácticas pertinentes que favorezcan la adquisición de conceptos matemáticos de manera significativa.

En muchos casos, los educadores y las educadoras que actualmente se encuentran enseñando matemáticas en las aulas de educación general básica pasaron por un sistema de enseñanza donde predominaba el enfoque tradicional.

Como consecuencia de lo anterior, y una posible causa de la problemática existente en 
torno a la enseñanza de la matemática, es que en la actualidad esta asignatura así como los conceptos y destrezas que involucra, carezca de significado para la mayoría del personal docente, por lo que imparten sus clases de la misma manera en que les fue enseñada, es decir, mecánicamente sin alcanzar la comprensión de cada proceso al momento de efectuar alguna operación matemática.

Por lo tanto, es necesario replantear el enfoque curricular predominante hasta el momento en la metodología empleada, ya que no es el alumno o la alumna quien debe estar en función del contenido, sino todo lo contrario; el currículo debe concebirse de tal manera que beneficie tanto a los grupos de estudiantes como a la sociedad en general.

Se debe tener presente que no toda la población estudiantil llega a comprender los contenidos matemáticos fijados en los currícula oficiales de la enseñanza obligatoria; a cierta parte de la población estudiantil se les dificulta, otros pierden el interés y esto evitará que se esfuercen lo suficiente, pero a todos les será necesario alcanzar cierto dominio en la utilización de conceptos básicos en matemática, pues resultan indispensables, tanto para su futura ocupación laboral como para su vida.

Se debe estimular la adquisición del conocimiento lógico matemático de manera que cada alumno sea capaz de descubrir la importante relación existente entre esta materia y la vida cotidiana.

\section{El conocimiento lógico matemático}

González y Jarillo (1994) explican que el conocimiento lógico matemático no se toma de los objetos sino de las acciones efectuadas sobre ellos. Es por medio de la acción que los objetos serán incorporados a los esquemas y estructuras de los sujetos; además, los sujetos no conocen más propiedades de las cosas que aquellas que su acción les permite conocer.
Se debe tomar en cuenta por tanto, que el conocimiento lógico matemático se acrecienta por medio de la relación con el entorno, de aquí deriva la importancia de propiciar en las clases de matemática momentos en los cuales los niños y las niñas puedan estar en contacto con material concreto proveniente del medio externo que los rodea. De esta forma, los alumnos y las alumnas serán capaces de comprender el sentido de la matemática al descubrir que esta se encuentra presente en todos los elementos del entorno, así como en las actividades que realizan, ya que la matemática no se encuentra solamente en el aula sino en cada una de las ocupaciones existentes.

Echenique (2006, p. 10) explica que se debe enseñar a los alumnos "a pensar matemáticamente, es decir, a que sean capaces de abstraer y aplicar ideas matemáticas en un amplio rango de situaciones $\mathrm{y}$, en este sentido, los propios problemas serán las "herramientas" que les llevarán a ello".

Es importante tener presente que la adquisición del conocimiento matemático va paralela al desarrollo del pensamiento lógico, y el eje central en torno al cual gira esta adquisición y desarrollo, es la resolución de problemas.

La resolución de problemas, es un aprendizaje que ha de realizarse a lo largo de la vida, contribuye a desarrollar en los niños y las niñas estrategias mentales básicas que les facilita resolver situaciones de la vida real, aplicando los conocimientos que se han adquirido durante los diferentes niveles educativos.

Ante esto, es necesario que las situaciones problemáticas que se le presenten a la población estudiantil, puedan ser relacionadas con la realidad que lo rodea, ya que le será más fácil a un estudiante pensar de manera lógica cuando es capaz de vivenciar el problema y de manipular objetos con el fin de lograr una mayor comprensión de la situación.

Es fundamental saber estimular el pensamiento lógico matemático mediante 
diversidad de actividades que tiendan a incrementar la creatividad, con la cual los niños y las niñas logren descubrir el conocimiento así como poner en práctica el acervo que ya poseen. Se debe reconocer que la lógica en matemática es una destreza que puede y debe ser desarrollada y fortalecida durante el periodo escolar.

La enseñanza de la matemática debe ser atendida con especial cuidado, con el fin de lograr que los niños y las niñas desarrollen sus habilidades correspondientes al periodo en que se encuentran.

\section{Enseñanza de la matemática}

La matemática es una materia que generalmente despierta sentimientos encontrados, hay quienes la aprecian, así como aquellos quienes después de haber terminado un año lectivo no quieren saber nada de ella; esto se debe principalmente por las experiencias o habilidades que haya tenido cada individuo. De este modo, algunas personas sienten frustración ante ejercicios o problemas matemáticos, y otros, por el contrario se sienten motivados y satisfechos al enfrentarse a esta disciplina.

El ambiente que se genere en los procesos de enseñanza y aprendizaje es un factor decisivo para propiciar ambos tipos de sentimientos; por ejemplo ante la falta de un ambiente propicio y de una metodología adecuada durante la enseñanza de la matemática, la experiencia resultante no podrá ser muy positiva.

El alumno será capaz de comprender de una mejor manera aquello que puede relacionar con sus experiencias, pues encontrará que posee mayor relevancia en $\mathrm{su}$ vida cotidiana. Lamentablemente esto no ocurre con frecuencia, ya que en su mayoría los docentes utilizan como eje central de la actividad matemática la pizarra y limitan la participación de sus estudiantes; por lo general, las actividades escolares fomentan la memorización y el estudiante no es capaz de poner en práctica la información que ha aprendido de memoria, o en la mayoría de los casos la olvida fácilmente después de resolver un examen.

De ahí que las estrategias de aprendizaje utilizadas adquieran tanta importancia durante el proceso de enseñanza. Con respecto a esto el Ministerio de Educación Pública (2005, p. 18) plantea que "las actividades y situaciones que se diseñen, tienen que enfocarse hacia la comprensión, asimilación e interiorización de conceptos de la matemática, a partir de la manipulación que el niño y la niña hagan de los materiales o recursos didácticos” (...).

Es fundamental para la enseñanza significativa de la matemática buscar el modo de conexión entre el aprendizaje nuevo con los conocimientos que ya posee y facilitar de esta manera la comprensión del nuevo aprendizaje. El docente no puede desechar los conocimientos previos de sus estudiantes como si fueran inútiles; por el contrario, debe destacarlos y aprovecharlos para fomentar la confianza en sí mismo al reconocer que la información que traen consigo es importante para el proceso de enseñanza.

Es bien sabido que dentro del aula escolar hay estudiantes con multiplicidad de habilidades y capacidades, y esto no debe ser una limitante en el aprendizaje de la matemática, por tanto, es responsabilidad del docente generar el ambiente propicio para desarrollar al máximo la capacidad de cada estudiante aunque no todos lleguen al mismo nivel.

En la enseñanza de la matemática se ha dejado de lado el pensamiento analítico y reflexivo, el cual ha sido substituido por la memoria y la mecanización generada principalmente por la repetición de ejercicios. Según explica Sánchez (2001), la enseñanza ha transcurrido en representaciones simbólicas que no han sido abordadas en forma concreta; de este modo las operaciones matemáticas se convierten en manipulaciones simbólicas, al no ser aprendidas por medio de la manipulación de materiales 
concretos, se obstaculiza la comprensión de los procesos matemáticos.

Con el fin de trabajar la comprensión lógica de los problemas matemáticos, es fundamental que cada educador tenga presente cuáles son las habilidades que debe fomentar durante sus clases.

\section{Desarrollo de habilidades intelectuales}

Es importante que la enseñanza de la matemática no se centre exclusivamente en la adquisición de conocimientos ya que esto constituye una de las principales preocupaciones en el trabajo docente, al tener que abarcar un plan determinado.

En múltiples ocasiones por esta preocupación se descuida el desarrollo de habilidades intelectuales que son indispensables para lograr la comprensión de los procesos matemáticos. El desarrollo de ciertas habilidades podrá propiciar que la persona sea competente en la materia, de este modo será capaz de comprender los contenidos y procesos básicos al momento que los interrelaciona de manera adecuada con situaciones de su vida diaria.

Las actividades realizadas en una clase de matemática deben contribuir al desarrollo de la capacidad de pensamiento del alumno, con miras a que cada individuo dentro del aula aprenda a razonar matemáticamente y aumente su capacidad para resolver problemas.

Con el desarrollo de ciertas habilidades, se busca además incrementar la inteligencia lógica matemática en la población estudiantil, la cual consiste en la facilidad para identificar diversidad de figuras geométricas, resolver operaciones numéricas complejas con facilidad y gusto, utilizar el pensamiento abstracto y solucionar problemas que pueda relacionar con la vida cotidiana.

Se reconoce en este aspecto la importancia de la matemática, no sólo para abarcar una serie de contenidos, sino para el desarrollo de destrezas útiles en la vida, con las cuales logren resolver, en un futuro, problemas de su propio entorno e incluso sean capaces de proponer soluciones ante los problemas sociales que deba enfrentar.

El programa de matemática que propone el Ministerio de Educación Pública para I ciclo de la educación general básica establece una serie de habilidades que deben ser desarrolladas con el fin de lograr la comprensión de la matemática. A continuación se explica cada una de ellas:

- Clasificación: esta habilidad es básica en la construcción de los diferentes conceptos matemáticos, como son los de número, y las operaciones numéricas. Esta habilidad se desarrolla en la medida en que el estudiante descubra por sí mismo los criterios de clasificación, no basta con que los clasifique a partir de un criterio dado.

- Flexibilidad del pensamiento: implica que el estudiante reconozca que un problema puede ser resuelto de diferentes maneras. El docente debe contemplar que en múltiples ocasiones los alumnos utilizan estrategias para resolver un problema o ejercicio sin que les hayan sido enseñadas.

- Estimación: es una habilidad que permite dar una idea aproximada de la solución de un problema o ejercicio, se desarrolla proponiendo al niño que dé respuestas aproximadas, lo cual permitirá tener una idea de lo razonable del resultado que obtenga. La estimación pone en evidencia el manejo que se tiene del sistema de numeración y el cálculo mental.

- Generalización: el desarrollo de esta habilidad permitirá al alumno generalizar relaciones matemáticas o estrategias de resolución de problemas.

- Imaginación espacial: esta habilidad implica que los alumnos 
desarrollen procesos que les permitan ubicar los objetos en un plano determinado, interpretar figuras tridimensionales, estimar longitudes, áreas o volúmenes.

- Reversibilidad del pensamiento: se refiere a seguir una secuencia en orden progresivo, al reconstruir procesos mentales en forma directa o inversa; es decir, que tengan la habilidad de hacer acciones opuestas simultáneamente (MEP, 2005). Por medio de esta acción los estudiantes no solo deben ser capaces de resolver un problema, sino de plantearlos a partir del resultado.

Es importante reflexionar sobre la dificultad que conlleva la tarea de enseñar a resolver problemas matemáticos y al mismo tiempo de la necesidad de desarrollar en los niños y las niñas las habilidades citadas anteriormente. Ante la escasa estimulación que se les da dentro de las aulas, surge una seria problemática, la cual se expone en el siguiente apartado.

\section{Problemática en torno a la metodología empleada}

Los métodos tradicionales empleados actualmente en la enseñanza de la matemática generan mayor desmotivación por parte de los estudiantes hacia la materia. La metodología influye en gran medida en la actitud que puedan presentar los estudiantes, por lo tanto, si el docente se preocupa por presentar el contenido de forma atractiva, será posible que sus alumnos muestren una actitud más positiva independientemente de su habilidad hacia la materia.

La práctica pedagógica refleja que en el sistema educativo costarricense existe gran preocupación por parte de los docentes por cumplir con cada uno de los contenidos y objetivos que propone el Ministerio de Educación Pública, esto genera que las estrategias didácticas utilizadas sean poco significativas, además adquiere gran relevancia el libro de texto, con lo cual el protagonismo del alumno disminuye.

Este primer hecho hace que la tarea de resolver problemas se torne más difícil, ya que por la prisa que ocasiona abarcar el currículo preescrito, no se le da el tiempo necesario al estudiante para que interiorice cada problema y trate de resolverlo por sí mismo. Además la resolución de problemas es vista como un tema más dentro del programa escolar, y al igual que todos los otros, se pretende asignarle un tiempo para ser estudiado, por lo que deja de ser el eje central de la matemática.

El docente debe desarrollar estrategias de aprendizaje que faciliten la comprensión, por lo que no debe abandonar en el libro de texto la conducción del trabajo del alumno, la interacción entre el docente y el estudiante es fundamental para el adecuado desarrollo del proceso de enseñanza de la matemática.

González y Jarillo (1994, citado en Terán y otros (2005, p. 172) afirma que "es condición necesaria y urgente, repensar la manera como se trabaja la matemática dentro de las aulas de la Escuela Básica”, esto porque generalmente esta disciplina es enseñada de una manera descontextualizada a los ámbitos de la vida real de los alumnos y las alumnas y sin ninguna relación con otras áreas curriculares.

Para que cada estudiante pueda encontrarle sentido a la matemática como una materia necesaria para la vida es fundamental la actuación del docente, quien tiene la responsabilidad de integrar en sus clases diversos aspectos cotidianos con el fin de hacer ver a sus estudiantes la significatividad de la materia. Además, es fundamental que exista una relación entre la matemática con las demás materias y de este modo hacer conscientes a los estudiantes de su importancia en todo plano de la vida.

Es necesario que las situaciones problemáticas planteadas al alumnado se 
presenten en contextos y situaciones reales de acuerdo con su entorno, su edad y las experiencias previas que posea. Por esta razón, el docente debe ver en el libro de texto un apoyo a su labor, ya que en ciertas ocasiones no se encuentra contextualizado al ambiente que vive el niño y la niña o los ejercicios y problemas no se ajustan a la capacidad que poseen, motivo que contribuye a acrecentar la falta de sentido en la materia.

Es fundamental conocer qué tipo de dificultades surgen como consecuencia de una enseñanza poco eficaz en la resolución de problemas con el fin de trabajar en la mejora del proceso de enseñanza aprendizaje de esta área. Si el docente no reconoce cuáles son las principales limitaciones que debe enfrentar no será capaz de mejorar su labor, pues primero se debe tomar conciencia sobre aquello que requiere un cambio.

Puig (1996, p. 22) explica que

...el profesor de matemáticas debería luchar por posibilitar que cada alumno desarrolle, dentro de sus capacidades, la comprensión y destrezas matemáticas exigidas para la vida adulta, para el trabajo y para posteriores estudios y aprendizajes, teniendo presentes las dificultades que algunos alumnos experimentarán para lograr una comprensión apropiada.

Con respecto a lo anterior, se evidencia la importancia de la enseñanza de la matemática, no sólo para abarcar una serie de contenidos, sino para el desarrollo de destrezas útiles en la vida.

De esta manera, la resolución de problemas matemáticos, debe aprovecharse para desarrollar en la población estudiantil destrezas básicas como la comprensión, el análisis, la creatividad... con las cuales logren en un futuro resolver problemas de su propia vida e incluso sean capaces de proponer soluciones ante los problemas sociales que deba enfrentar. De ahí la importancia de la resolución de problemas matemáticos.

\section{Resolución de problemas matemáticos}

Los alumnos deben construir conceptos matemáticos a partir de la resolución de problemas, ya que esta habilidad les permite hallar la relación entre la matemática y su vida. Ruiz y otros (2003, p. 325) explican que la resolución de problemas se concibe "como generadora de un proceso a través del cual quien aprende combina elementos del conocimiento, reglas, técnicas, destrezas y conceptos previamente adquiridos para dar solución a una situación nueva". Así, la resolución de problemas puede considerarse como el eje central de la enseñanza en matemática.

De acuerdo con Buschiazzo y otros (1997, p. 58) desde el punto de vista matemático, "el problema implica una dificultad, ya que se plantea una situación nueva que se debe dilucidar por medio del razonamiento. La superación de esta dificultad que se habrá de alcanzar a través de algún camino constituye la resolución del problema”.

A partir de lo anterior se reconoce que el problema debe conformar un reto para el alumno, y debe ser adecuado al nivel de formación de cada grupo. De este modo, no se puede pretender que todos en el aula resuelvan siempre los mismos problemas; al considerar que dentro de una misma clase se pueden encontrar estudiantes con distintas capacidades cognitivas, es lógico que resuelvan problemas con diferentes niveles de dificultad, de manera que constituya un verdadero reto para todos.

Un problema no se debe responder de manera inmediata, lo cual no quiere decir que la situación planteada debe estar más allá de lo que podría resolver el niño o la niña de acuerdo a su etapa de desarrollo cognitivo; sino que la situación que se le presenta no es idéntica a alguna que haya resuelto anteriormente por lo que amerita un esfuerzo mayor para comprenderla. Normalmente, cuando los alumnos se enfrentan a un reto así, no son capaces de 
resolverlo, no porque no puedan, sino porque no están acostumbrados a hacerlo y no están conscientes que requiere mayor participación de su parte por lo que se dejan vencer fácilmente.

Es importante que los alumnos y alumnas sean capaces de explicar y justificar el proceso seguido en la resolución de problemas y comprendan la razón de las soluciones que proponen, es necesario que entiendan por qué ciertos procedimientos conducen a la respuesta esperada y otros no.

Según establecen Ruiz y otros (2003, p. 327) "dejar hablar [a] los niños sobre sus acciones, permite al maestro acceder a su pensamiento". De ahí que se valore el brindar espacios en los cuales se dé la oportunidad para expresarse oralmente y así conocer cómo piensan el niño y la niña; con el fin de poder entender su pensamiento y de este modo utilizar la mejor estrategia para guiarles hacia la búsqueda de una solución eficaz del problema. En este proceso es fundamental tomar en cuenta los distintos ritmos de aprendizaje, ya que muchos alumnos necesitarán más tiempo de discusión y trabajo oral, antes de poder abordar por escrito los problemas.

Es necesario evitar la enseñanza de métodos mecánicos para resolver un problema, por lo general los docentes acuden a un único procedimiento para resolverlos y no dejan libertad de pensamiento a sus estudiantes, quienes deben utilizar la misma estrategia de solución que le fue enseñada. Por el contrario, se debe impulsar a cada estudiante a hallar la solución del problema por sí mismo, debe ser capaz de reconocer que existe más de una forma para darle solución y no limitarse a una única manera de hacerlo.

El profesor debe ayudar a los alumnos y alumnas a comprender la mejor forma en que pueden aplicar los conocimientos o destrezas que posean o estén aprendiendo y cómo han de hacer uso de ellas en la resolución de problemas. Para facilitar la utilización de las destrezas y habilidades adquiridas, los problemas han de guardar relación tanto con situaciones cotidianas como con otras menos familiares.

Según Buschiazzo y otros (1997, p. 74) "la resolución del problema no debe involucrar cálculos largos, que hagan fatigar y al cabo de los cuales se pueda perder el hilo del razonamiento y aún el interés por su resolución”. El docente debe plantear problemas que supongan verdaderos retos para sus estudiantes; es importante que los problemas tengan una presentación diversa para evitar la mecanización, pues por lo general los alumnos no buscan comprenderlos sino identificar el tipo de operación al que hace referencia el problema.

Los niños y las niñas deben enfrentarse al problema de manera creativa y ser ellos quienes propongan la mejor forma de solución, para esto es necesario que estén en contacto con el material necesario que les permita apoyar su pensamiento. Además, es fundamental que utilicen el conocimiento que poseen para generar uno nuevo que les permita comprender la información relevante para resolver el problema.

Será muy probable que cometan errores al intentar solucionarlo pero no se debe ver como falta de inteligencia, por lo que es importante analizar el procedimiento seguido para detectar las razones que le han impedido tener éxito.

Comúnmente en las clases de matemática se observa que los estudiantes olvidan con frecuencia lo que deben hacer cuando se retoma un contenido que ha sido visto anteriormente. Esto se da principalmente por su falta de intervención en el proceso de resolución de ejercicios y problemas; por tanto, es necesario incentivar la participación de los niños y las niñas como sujetos del proceso enseñanza aprendizaje, cada estudiante debe reconocer su capacidad de pensamiento y determinar sus avances y errores en el transcurso del aprendizaje.

Ante la resolución de problemas los alumnos deben reflexionar sobre la situación y las acciones que realizan en el proceso, es fundamental una actitud crítica ante el propio trabajo y el de los demás. 
Los niños deben ser partícipes activos en el proceso de enseñanza, para lo cual se deben planear diversas actividades que sean atractivas e interesantes con el fin de motivarlos a participar con entusiasmo.

Es fundamental la existencia de un ambiente en el cual se tolere la reflexión, la duda, la exploración y la discusión sobre las diferentes maneras de comprender una misma situación problemática. Crear un clima de tranquilidad es fundamental para facilitar la concentración a la hora de plantear el problema y evitar así que los estudiantes se precipiten a resolver el problema muchas veces de manera errónea, por el simple hecho de terminarlo.

Buschiazzo y otros (1997) explican ciertas tareas importantes que posee el docente en la enseñanza de resolución de problemas, entre las cuales se destacan:

- Selección de problemas: para esto el docente debe tener en cuenta las características del grupo en general con el fin de contextualizar la situación problemática; además debe contemplar las características individuales, para adecuar el problema al nivel cognitivo de sus estudiantes.

- Orientar la resolución: el educador debe actuar como guía en la resolución del problema, debe permitir que sea el estudiante quien proponga las soluciones y se dé cuenta de sus errores. Esto no quiere decir que el docente se muestre como un simple espectador, sino que oriente el proceso de manera que evite dar una única ruta de solución a sus alumnos.

- Estimular la resolución de problemas: será común que en el proceso los estudiantes sientan desánimo ante la dificultad que se les presente, ante esto el educador debe motivarlos para que muestren una actitud positiva en todo momento.

- Debe ser modelo ante la resolución de problemas: mediante la actitud que tenga, el docente puede transmitir una serie de sentimientos a sus estudiantes; por lo que es indispensable que sea optimista $\mathrm{y}$ muestre gusto ante los problemas que se están resolviendo. Por tanto debe evitar comentarios o gestos que puedan desanimar a los niños o a las niñas.

Para lo anterior es relevante la tarea del educador o la educadora, quien debe preparar sus clases de manera adecuada para facilitar la enseñanza a sus estudiantes; dentro de las particularidades del proceso de enseñanza y aprendizaje de la matemática, al igual que en cualquier área del conocimiento, uno de los elementos claves del mismo viene a estar representado por la figura del docente el cual en su condición de mediador, deberá estar preparado y capacitado para convertir el ambiente educativo en un laboratorio de experiencias que conduzca a cada estudiante a crear y construir su conocimiento utilizando al máximo sus habilidades de acuerdo con su nivel de desarrollo.

Cada una de las funciones mencionadas son necesarias para incentivar la búsqueda de soluciones por parte del alumnado. Para lograrlo, se debe estimular el razonamiento mediante la aplicación de diversos métodos ante una misma situación.

\section{Métodos de resolución de problemas}

Se debe tener claro que no existen recetas mágicas para dar solución a un problema, pero se pueden utilizar ciertos pasos que son esenciales para hacerlo, en los siguientes párrafos se explican algunos métodos que facilitan este proceso.

Echenique (2006, p. 23) aclara que

Durante muchos años y todavía en nuestros días, la mayor parte de los problemas matemáticos que se proponen en clase tienen como finalidad aplicar los contenidos o algoritmos que se han estudiado en la unidad didáctica de la que forman parte. 
Estas actividades no potencian la búsqueda de procedimientos de resolución, sino que, más bien al contrario, a menudo se presentan como baterías de problemas que los alumnos resuelven de forma mecánica.

Generalmente se les pide que los trabajen de forma individual, por lo que no discuten las razones por las que lo resolvieron de esa manera o por qué aplicaron cierto algoritmo, en otros casos se dejan como tareas y se revisan al día siguiente sólo las respuestas. De este modo los estudiantes no son capaces de salir de la mecanización; para evitarlo se proponen algunos métodos de los cuales el docente se puede valer para impartir sus clases de manera más significativa.

1. Polya (1949), citado por Echenique (2006), establece cuatro etapas en la resolución de un problema:

- Comprender el problema: implica entender tanto el texto como la situación que presenta el problema, diferenciar los distintos tipos de información que ofrece el enunciado y comprender qué debe hacerse con la información que es aportada. Se debe leer el enunciado despacio, tratando de contestar las siguientes interrogantes:

¿Cuáles son los datos? (lo que conocemos).

¿Cuáles son las incógnitas? (lo que buscamos).

Después hay que tratar de encontrar la relación entre los datos y las incógnitas y si es posible, se debe hacer un esquema o dibujo de la situación.

- Diseñar un plan: es la parte fundamental del proceso de resolución de problemas. Una vez comprendida la situación planteada y teniendo clara cuál es la meta a la que se quiere llegar, es el momento de plani- ficar las acciones que llevarán a ella, es necesario abordar cuestiones como para qué sirven los datos que aparecen en el enunciado, qué puede calcularse a partir de ellos, qué operaciones utilizar y en qué orden se debe proceder.

- Ejecución del plan: consiste en la puesta en práctica de cada uno de los pasos diseñados en la planificación. Es necesaria una comunicación y una justificación de las acciones seguidas: primero calculo..., después..., por último... hasta llegar a la solución. Esta fase concluye con una expresión clara y contextualizada de la respuesta obtenida.

- Examinar la solución: es conveniente realizar una revisión del proceso seguido, para analizar si es o no correcto el modo como se ha llevado a cabo la resolución. Es preciso contrastar el resultado obtenido para saber si efectivamente da una respuesta válida a la situación planteada, reflexionar sobre si se podía haber llegado a esa solución por otras vías, utilizando otros razonamientos.

Las etapas anteriormente mencionadas, normalmente no se dan dentro de las aulas y son indispensables para conocer el modo de pensar, razonar y actuar de los estudiantes y de esta forma ayudarlos a corregir sus errores.

2. Pinteño y otros (1999) establecen que la presentación de un tema matemático debe estar basada en la resolución de problemas, de este modo se pone énfasis en los procesos de pensamiento de los estudiantes. De acuerdo con De Guzmán (2000 citado por Pinteño y otros, 1999, p. 207), 
la forma de presentación de un tema matemático basada en el espíritu de la resolución de problemas debería proceder más o menos del siguiente modo:

1. Propuesta de la situación problema de la que surge el tema (basada en la historia, aplicaciones, modelos, juegos...).

2. Manipulación autónoma por el alumnado.

3. Familiarización con la situación y sus dificultades.

4. Elaboración de estrategias posibles.

5. Ensayos diversos por el alumnado.

6. Herramientas elaboradas a lo largo de la historia (contenidos motivados).

7. Elección de estrategias.

8. Ataque y resolución de los problemas.

9. Generalización.

10. Nuevos problemas.

En todo el proceso el eje principal ha de ser la propia actividad dirigida por el profesorado, colocando al alumnado en situación de participar, sin aniquilar el placer de ir descubriendo por sí mismo lo que los grandes matemáticos y matemáticas han logrado con tanto esfuerzo.

3. Ruiz y otros (2003), en su investigación, diseñaron y aplicaron estrategias para promover la construcción de aprendizajes de contenidos matemáticos relacionados con la lectura, la escritura y el juego. En esto destacó algunos elementos importantes para la enseñanza de conceptos matemáticos, localizados en la revisión de literatura:

Explorar los conceptos numéricos desarrollados por los niños en forma natural con la finalidad de reconocer las reglas implícitas seguidas por éstos y construir sobre ellas otros conceptos numéricos (Resnick,1989; Kamii, 1994, citado en Ruiz y otros, 2003, p. 323).

Promover la resolución de problemas como estrategia didáctica que permite el desarrollo del razonamiento autónomo, al posibilitar la construcción y reconstrucción de soluciones (Resnick, 1989; Kamii, 1994; Ministerio de Educación, 1997, citado en Ruiz y otros, 2003, p. 323).

Promover la comunicación oral y escrita, como forma de hacer con las palabras las mismas acciones que se hacen con los objetos, a fin de desarrollar la internalización de las acciones externas y la capacidad lingüística al sustentar afirmaciones usando diversas formas argumentativas, desde el lenguaje común hasta el lenguaje formal (...).

Propiciar el desarrollo de la "reversibilidad", como estrategia cognitiva, mediante la cual en la acción de "devolverse" debe lograrse la comprensión de las nuevas relaciones que aparezcan y de la forma diferente en que se manifiestan las acciones preliminares (...).

Considerar la importancia de la actividad lúdica, lo cual implica ofrecer [a cada estudiante] la oportunidad de construir y reconstruir la realidad con la ayuda de instrumentos simbólicos y reglas, mediante los juegos. En el juego se realizan los mayores logros del [educando] (Ruiz, 2003, p. 323).

Quizás el método más sencillo de aplicar sea el propuesto por Polya (1949), citado por Echenique (2006), a partir de este, el docente puede ser capaz de integrar aspectos que resaltan los otros modelos. En página siguiente se da un ejemplo concreto de cómo se puede aplicar en una clase de resolución de problemas.

Los métodos citados pueden constituir el punto de partida hacia el mejoramiento de la enseñanza de la resolución de problemas en matemáticas, no se trata que el docente los siga al pie de la letra, mucho menos que elija solamente uno para aplicarlo por el resto de su vida.

La idea central es que tome de cada uno lo que más le convenga según sus necesidades, siempre y cuando propicie la participación activa de los niños y las niñas y tenga presente que son ellos y ellas quienes deben hallar la estrategia de resolución.

Es fundamental que los métodos que se proponen sean aplicados desde la etapa inicial de la escolaridad, ya que se deben formar desde pequeños y de este modo los problemas y el enfrentarse a ellos sea visto con naturalidad y parte del trabajo cotidiano en el aula. 
Objetivos:

- Aplicar las cuatro fases del método de resolución de problemas de George Polya.

- Analizar cada una de las acciones que se llevan a cabo para resolver un problema.

\begin{tabular}{|c|c|}
\hline Primer momento & $\begin{array}{l}\text { Presentación del problema: conviene que al iniciar el problema sea sencillo, luego se } \\
\text { pasa a otros problemas con mayor grado de dificultad. }\end{array}$ \\
\hline Segundo momento & $\begin{array}{l}\text { Comprensión del problema: } \\
\text { - } \quad \text { Se debe leer el enunciado despacio. } \\
\text { - } \quad \text { Determinar ¿cuáles son los datos que conocemos? } \\
\text { - } \quad \text { ¿Cuáles son las incógnitas? (lo que buscamos). } \\
\text { - } \quad \text { Hay que tratar de encontrar relación entre los datos y las incógnitas. } \\
\text { - } \quad \text { Si se puede, se debe hacer un dibujo o esquema que aclare la situación. }\end{array}$ \\
\hline Tercer momento & $\begin{array}{l}\text { Diseño del plan: } \\
\text { Se pueden formular algunas preguntas generadoras. } \\
\text { - ¿Se parece el problema a otros que ya conocemos? } \\
\text { • ¿Es posible plantear el problema de otra forma? } \\
\text { - } \quad \text { Imaginar un problema parecido pero más sencillo, se puede relacionar con casos } \\
\quad \text { de la vida real. } \\
\text { ¿Son necesarios todos los datos? }\end{array}$ \\
\hline Cuarto momento & $\begin{array}{l}\text { Ejecución del plan: } \\
\text { - Se debe comprobar cada uno de los pasos. } \\
\text { - } \quad \text { Antes de hacer algo se debe pensar: ¿Qué se consigue con esto? } \\
\text { - Se debe acompañar cada operación matemática de una explicación, contando lo } \\
\text { que se hace y para qué se hace }\end{array}$ \\
\hline
\end{tabular}

\section{A manera de conclusión}

No basta con presentar problemas matemáticos para que los educandos los resuelvan. Es necesario darles un tratamiento adecuado, analizando las estrategias y técnicas de resolución utilizadas, se debe dar oportunidad a cada estudiante de expresarse para conocer su modo de pensar ante las diversas situaciones que se le presentan.

Cada docente debe promover la asimilación e interiorización de conocimientos matemáticos en sus estudiantes, con el fin de que adapten esos conocimientos para resolver problemas que no les sean tan habituales, así como para plantearse otras cuestiones a partir de ellos.

En este sentido, los modelos de resolución de problemas ocupan un papel importante pues son fundamentales para el mejoramiento de la enseñanza de los mismos, para aplicarlos se debe dedicar un espacio en el horario escolar y conseguir un clima propicio en el aula que favorezca la adquisición de destrezas. Si bien es cierto, el aplicar algún método conlleva más tiempo del que se acostumbra dedicar normalmente a la resolución de problemas; no se debe tomar como pérdida de tiempo, pues durante el proceso cada estudiante será capaz de adquirir mayor comprensión y habilidades intelectuales necesarias para toda su vida.

Se debe tener presente que la matemática no se aprende por transmisión directa de lo que explica el docente o de la información que se obtiene de los libros de texto; sino que se aprende en interacción con situaciones problemáticas las cuales obligan al estudiante a modificar su estructura cognitiva por el contacto con una multiplicidad de acciones que requieren distintas habilidades. 


\section{Referencias bibliográficas}

Buschiazzo, N., Cattáneo, L., Filipputti, S., Hinrichsen, S. y Lagreca, N. (1997). Matemática hoy en la E.G.B.: ¿qué enseñar? ¿cómo? ¿para qué?. Estrategias didácticas. Rosario: Homo Sapiens Ediciones.

Castillo, L. (2003). Enfoques o concepciones curriculares. Instituto Profesional de Providencia. Escuela de Educación. Educación Básica. [Resumen] (s.p.). Santiago, Chile. Extraído el 3 de marzo de 2003 de

http://www.asesoríaspedagogicas.cl/ ipp/clase.curriculum.doc

Echenique, I. (2006). Matemáticas resolución de problemas. Educación Primaria. Navarra: Departamento de Educación. Gobierno de Navarra. Extraído el 25 de abril de 2007 de http://www.pnte.cfnavarra.es/ publicaciones/pdf/matematicas.pdf.

González, L. y Jarillo, R. (1994). La enseñanza y el aprendizaje de la matemática en la educación básica: un enfoque constructivista. Costa Rica: Ar-Lit-o.

Ministerio de Educación Pública [MEP]. Dirección Desarrollo Curricular. (2005). Programas de estudio. I y II Ciclo: Matemática I ciclo. Costa Rica. Extraído el 11 de octubre de 2007 de http://www.mep.go.cr/Curricular/ index.aspx

Ministerio de Educación Pública [MEP]. Dirección Desarrollo Curricular. (2005). Programas de estudio. I y II Ciclo: Matemática II ciclo. Costa Rica. Extraído el 11 de octubre de 2007 de http://www.mep.go.cr/Curricular/ index.aspx

Pinteño, A. [Coord.], Alcalá, A., Mesa, P., Alfaro, V., Martínez, J., Navarro, J. y otros. (1999). Mejora del rendimiento en el Área de Matemáticas a través de la resolución de problemas con alumnado de Educación Primaria. Extraído el 11 de octubre de 2006 de http://www.juntadeandalucia.es/ averroes/publicaciones/investigacion/ rendimiento_mat.pdf -

Programa Estado de la Nación en Desarrollo Humano Sostenible. (2005). CONARE [Coord.]. Informe Estado de la Educación Costarricense (1). San José, Costa Rica: Programa del Estado de la Nación.

Puig, L. (1996). Investigación y didáctica de las matemáticas. Madrid: Ministerio de Educación y Ciencia.

Ruiz, D. y García, M. (2003, OctubreDiciembre). El lenguajecomo mediador en el aprendizaje de la aritmética en la primera etapa de Educación Básica. Educere La Revista Venezolana de Educación, 23(7): 321- 327.

Sánchez, L. M. (2001). Dificultades de los alumnos de sexto grado de educación primaria para la resolución de problemas matemáticos. Análisis retrospectivo. Tesis de Maestría en Ciencias para la obtención del título de Maestra en Ciencias. Facultad de Ciencias de la Educación. Área: Investigación Educativa. Universidad de Colima, México. Extraído el 25 de setiembre de 2006 de http:// www.digeset.ucol.mx/tesis_posgrado/ indic1.php

Terán, M. y Pachano, L. (2005, AbrilJunio). La investigación-acción en el aula: tendencias y propuestas para la enseñanza de la matemática en sexto grado. Educere La Revista Venezolana de Educación, 029(9): 171-179. 